\title{
Oficinas de Gestão do Tempo com Estudantes Universitários
}

\author{
Clarissa Tochetto de Oliveira \\ Universidade Federal do Rio Grande do Sul, RS, Brasil. \\ Marco Antônio Pereira Teixeira \\ Universidade Federal do Rio Grande do Sul, RS, Brasil.
}

\author{
Rodrigo Carvalho Carlotto \\ Universidade Federal de Santa Maria, RS, Brasil. \\ Ana Cristina Garcia Dias \\ Universidade Federal de Santa Maria, RS, Brasil.
}

Resumo: Buscou-se descrever a experiência de oficinas de gestão do tempo realizadas junto a acadêmicos de uma universidade pública no interior do Rio Grande do Sul. Foram realizados oito encontros com o objetivo de avaliar e discutir a forma como os participantes costumam organizar o seu tempo. Participaram 85 alunos de graduação e pós-graduação. Os tópicos abordados incluíram estratégias utilizadas para organização, identificação de problemas, metas e objetivos e oferecimento de dicas. Foram descritos os procedimentos e as ferramentas utilizadas nas oficinas. As dificuldades vivenciadas pelos estudantes estavam relacionadas à procrastinação, à incapacidade de dizer "não" às demandas alheias, à carga horária de seus cursos e à conciliação entre estudos, convivência familiar e lazer. A participação na oficina foi um primeiro momento de reflexão sobre a temática para muitos participantes. Pesquisas que investiguem os "motivos" das dificuldades na gestão do tempo nessa população podem subsidiar futuras intervenções junto a esse público.

Palavras-chave: Tempo, Estudantes, Universidades.

\section{Workshops on Time Management With College Students}

\begin{abstract}
The aim of this study was to describe the experience of workshops on time management with college students from a university from Rio Grande do Sul. Eight workshops were held in order to evaluate and to discuss the way students usually organize their time. Eighty five undergraduate and graduate students took part of the workshops. The topics included planning strategies, identification of problems, goals, and objectives, and tips. Procedures and tools used in the meetings were described. The difficulties faced by students were related to procrastination, inability to say no to others' demands, workload of their courses, and management of study, family, and leisure. The participation in the workshops was the first opportunity for students to think about the subject. New research investigating why students get into time management difficulties might contribute to future interventions.

KeYwords: Time management, Students, Universities.
\end{abstract}


De Olineira, Clarissa Tochetto; Carlotto, Rodrigo Carvalho; Teixeira, Marco Antônio Pereira;

Dias, Ana Cristina Garcia (2015). Oficinas de Gestão do Tempo com Estudantes Universitários.

\title{
Talleres de Gestión del Tiempo con Estudiantes Universitarios
}

\begin{abstract}
Resumen: Se buscó describir la experiencia de talleres de gestión del tiempo realizados junto con estudiantes de una universidad del interior de Río Grande del Sur. Se han llevado a cabo ocho encuentros con el objetivo de evaluar y discutir la manera como los participantes suelen organizar su tiempo. Han participado 85 alumnos de pregrado y posgrado. Los temas abordados incluyeron estrategias utilizadas para la organización, identificación de problemas, metas y objetivos, así como para ofrecimiento de sugerencias. Se han descrito los procedimientos y herramientas utilizados en los talleres. Las dificultades vividas por los estudiantes estuvieron relacionadas con la dilación, la incapacidad de decir "no" a las demandas ajenas, la carga horaria de sus carreras, y la conciliación entre estudios, convivencia familiar y ocio. La participación en el taller fue un primer momento de reflexión acerca de la temática para muchos participantes. Estudios que investiguen las razones de las dificultades en la gestión del tiempo de esa población pueden subsidiar futuras intervenciones junto a ese público.

Palabras clave: Gestión del tiempo, Estudiantes, Universidades.
\end{abstract}

Administrar o tempo de forma adequada constitui-se em uma necessidade do século XXI. Entretanto, especialistas afirmam que boa parte da população organiza de forma inadequada seus horários e compromissos (Barbosa, 2011). Não é raro que tarefas cujo prazo está distante ou que não possuem um prazo definido sejam negligenciadas pelos indivíduos. Uma das razões para isso é a tendência a dedicarmos maior atenção para atividades mais simples e com mais vantagens em curto prazo do que para outras, mais complexas e com benefícios obtidos somente a longo prazo (König, \& Kleinmann, 2007). Dessa forma, organizar as responsabilidades imediatas e conciliar o tempo com os planos futuros é um desafio enfrentado por muitos adultos na atualidade.

Na busca de soluções adequadas para este desafio, há estudiosos dedicados à pesquisa da administração do tempo (Covey, 2002). A partir disso, surgiram diferentes linhas de pensamento que, em geral, apresentam princípios básicos similares: organização, controle e priorização. A essência das ideias na área de gestão do tempo pode ser capturada em uma única frase: organize e execute conforme a prioridade. Esta frase representa a evolução de quatro gerações da teoria de gestão do tempo, e a melhor maneira de realizar isso é assunto para uma variedade de abordagens e métodos (Covey, 2002). A primeira geração caracterizou-se pela criação de bilhetes e listas, em um esforço de sistematizar as demandas feitas sobre o tempo e energia das pessoas. Já a segunda geração caracterizou-se pelo uso de calendários e agen- das, refletindo a tentativa de marcar eventos e atividades no futuro. Por sua vez, a terceira geração adiciona às gerações anteriores a ideia de prioridade, metas, planejamento diário e elaboração de plano para conquista dessas metas e atividades. Recentemente, emergiu uma quarta geração, na qual o norte-americano Stephen Covey se destaca pela proposição da matriz de Gerenciamento do Tempo. Dentro dessa perspectiva, o autor distribui as atividades em quatro quadrantes, conforme critérios de importância e urgência. Afirma que uma atividade pode ser: urgente e importante; não urgente e importante; urgente e não importante; não urgente e não importante (Covey, 2002).

De forma geral, a gestão do tempo consiste em uma série de hábitos ou comportamentos que implicam no uso efetivo do tempo para auxiliar na produtividade e reduzir o estresse. Esses comportamentos podem ser adquiridos através da experiência de vida, de treinamentos ou da prática (Claessens, Van Eerde, Rutte, \& Roe, 2007; MacCann, Fogarty, \& Roberts, 2012). Uma característica da gestão do tempo refere-se ao planejamento, ou seja, o indivíduo necessita decidir quais tarefas irá desempenhar, definir a prioridade de cada uma e lidar com as possíveis distrações na execução das mesmas para organizar seu tempo (Claessens et al., 2007). Assim, diversas competências estão envolvidas no desenvolvimento de uma boa gestão do tempo, tais como a definição de objetivos, estabelecimento de planos, a composição de listas de ati- 
vidades, o manejo de imprevistos e o cumprimento de prazos (Roberts, Schulze, \& Minsky, 2006).

A capacidade de organizar as atividades de acordo com o tempo disponível é um fator importante para o sucesso acadêmico de estudantes universitários (MacCann et al., 2012). Discentes com experiência de insucesso nesse nível de ensino, geralmente, investem pouco tempo e esforço no seu estudo pessoal (Rosário, Nunes, Magalhães, Rodrigues, Pinto, \& Ferreira, 2010). Uma das formas de planejar as atividades de estudo é estabelecer objetivos e critérios para avaliar até que ponto os mesmos foram alcançados. Para tanto, os estudantes podem considerar o ritmo pessoal de trabalho, os horários mais apropriados para o estudo, a quantidade de disciplinas que devem ser contempladas, o intervalo a ser observado no estudo de duas disciplinas, o tempo para descanso e a utilização de agenda (Figueiredo, 2008). A habilidade de organizar as atividades de acordo com o tempo disponível também pode ser considerada uma vantagem competitiva no mercado de trabalho (Claessens et al., 2007). O planejamento das atividades diárias, o estabelecimento de prioridades e de metas a longo prazo estão associadas com a produção e com o aproveitamento de ideias úteis (Zampetakis, Bouranta, \& Moustakis, 2010).

Entretanto, a administração do tempo pode constituir uma das vivências que gera maior dificuldade quando o estudante ingressa no ensino superior, pois ele deve lidar com uma série de exigências acadêmicas e administrativas (Basso, Graf, Lima, Schmidt, \& Bardagi, 2013; Sampaio, 2011). A dificuldade em planejar e cumprir atividades dentro do prazo pode ser ainda maior para os estudantes que trabalham, visto que precisam conciliar as atividades exigidas no trabalho, nos estudos e em outros contextos presentes em suas vidas (MacCann et al., 2012; Sarriera, Paradiso, Schütz, \& Howes, 2012). Além disso, problemas na gestão do tempo podem contribuir para a diminuição da autoeficácia na gestão acadêmica, especialmente quando as estratégias utilizadas pelos alunos para planejar e respeitar os prazos das tarefas exigem um esforço maior do que o esperado e resultam em retorno menor do que o idealizado (Guerreiro-Casanova, \& Polydoro, 2011).

A gestão do tempo no contexto educacional vem ganhando cada vez mais importância na atualidade, uma vez que a educação formal exige mais autonomia por parte das pessoas, e treinamentos e educação continuada ao longo de toda a vida são cada vez mais comuns. A flexibilidade das opções de estudo, marcada pela crescente substituição das aulas ao vivo por aulas virtuais, também exige que o aluno assuma uma postura ativa na gestão do tempo, visto que deverá definir o seu próprio ritmo de estudos e aulas. Assim, os estudantes que não conseguirem administrar seu tempo de forma eficaz podem enfrentar dificuldades ao longo do ensino superior (MacCann et al., 2012).

Os hábitos e comportamentos relacionados à gestão do tempo podem ser treinados em indivíduos que não os desempenham automaticamente (MacCann et al., 2012). Em um estudo realizado com trabalhadores holandeses, constatou-se que a participação dos mesmos em oficinas de gestão do tempo não só aumentou suas habilidades em administrar o tempo disponível, mas também diminuiu suas preocupações e hábitos de procrastinação (Van Eerde, 2003). Programas dedicados a melhorar a administração do tempo também têm sido desenvolvidos para professores de ensino médio, diretores e estudantes universitários. A realização desse tipo de oficina pode melhorar o sucesso acadêmico, especialmente para discentes que possuem dificuldades no desempenho dessa habilidade (MacCann et al., 2012).

O objetivo deste artigo consiste em descrever uma experiência com oficinas de gestão do tempo realizadas com estudantes universitários de uma universidade pública no interior do Rio Grande do Sul. As oficinas buscavam incentivar os participantes a avaliar a forma como costumam se organizar, bem como estimular o planejamento pessoal, apresentando estratégias alternativas.

\section{Método}

O projeto de extensão "Oficinas de Orientação Profissional e Planejamento de Carreira” oferece oficinas para estudantes universitários com o objetivo de contribuir para o desenvolvimento de habilidades relevantes para o planejamento de suas carreiras. Dentre as oficinas oferecidas nos anos de 2011 e 2012, oito abordaram o tema da Gestão do Tempo. A iniciativa de oferecer tais atividades surgiu a partir das principais dificuldades apresentadas pelos estudantes nos atendimentos psicoterápicos realizados pelo setor de apoio aos discentes da universidade, além de ser um problema frequente enfrentado pelos acadêmicos ao longo de seus percursos universitários (Soares, Poubel, \& Mello, 2009).

A divulgação das oficinas foi realizada através da página virtual da universidade e da página do setor de apoio ao estudante da instituição e do projeto em uma 


\section{De Oliveira, Clarissa Tochetto; Carlotto, Rodrigo Carvalho; Teixeira, Marco antônio Pereira; Dias, Ana Cristina Garcia (2015). Oficinas de Gestão do Tempo com Estudantes Universitários.}

rede social da internet. Os universitários interessados em participar entraram em contato através do correio eletrônico para ter o seu nome na lista de participantes. Também houve demanda pela oficina por parte de grupos fechados de estudantes universitários, como no caso de ONGs ou PETs. Nestes casos, um discente representante da ONG ou PET solicitou a realização da oficina específica para esses grupos. Assim, os participantes das oficinas eram estudantes que se inscreveram individualmente nas datas divulgadas e estudantes membros das ONGs ou PETs que solicitaram a realização das oficinas.

No total, 85 universitários participaram das oito oficinas oferecidas (sendo 11 participantes na primeira, 12 na segunda, 12 na terceira, 14 na quarta, 6 na quinta, 11 na sexta, 7 na sétima e 12 na oitava). A idade dos estudantes variou de 15 a 54 anos $(M=22,8$, $D P=5,4)$. Os participantes eram alunos de Administração, Agronomia, Ciências Biológicas, Ciências Contábeis, Ciências Sociais, Desenho Industrial, Direito, Economia, Educação Especial, Educação Física, Engenharia Elétrica, Engenharia Florestal, Engenharia Mecânica, Engenharia Química, Filosofia, Física, Fisioterapia, Fonoaudiologia, História, Informática, Jornalismo, Letras, Matemática, Medicina Veterinária, Meteorologia, Música, Odontologia, Produção Editorial, Psicologia, Publicidade e Propaganda, Química, Relações Internacionais, Relações Públicas, Tecnologia em Alimentos, Tecnologia em Fabricação Mecânica, Tecnologia em Geoprocessamento e Terapia Ocupacional. Dos estudantes que compareceram nas oficinas, 22 alunos $(25,88 \%)$ estavam cursando os semestres iniciais (até o terceiro semestre), 31 $(36,47 \%)$ os semestres intermediários (do quarto ao sexto semestre) e 17 (20,0\%) os semestres finais (do sexto ao décimo semestre). As oficinas ainda contaram com a presença de 10 alunos (11,77\% dos 85$)$ de pós-graduação em Comunicação, Educação em Ciências, Geografia, Psicologia e Química, dois $(2,35 \%)$ do pré-vestibular da universidade, dois $(2,35 \%)$ do colégio politécnico e de uma $(1,18 \%)$ professora do PET.

Para cada oficina, foram abertas 20 vagas, a fim de assegurar a efetividade das atividades desenvolvidas e de dar espaço para a participação dos envolvidos. As vagas foram preenchidas conforme a ordem de inscrição dos interessados (feita por correio eletrônico). As oficinas possuíam formato de encontro único e foram elaboradas e ministradas por estudantes de graduação e pós-graduação em Psicologia, orientados por um professor do mesmo curso.
Os encontros aconteceram nas dependências da própria universidade em horários que possibilitassem a participação de alunos com aulas diurnas e noturnas, com duração aproximada de 90 minutos. Durante as oficinas, solicitou-se aos participantes que preenchessem uma ficha informando o sexo, a idade, o curso e o semestre em que se encontravam para o mapeamento do perfil do grupo de trabalho. O material foi recolhido e guardado de modo a manter o sigilo sobre a identidade de cada participante.

As oficinas pretendiam promover a autoavaliação da forma como os participantes costumavam se organizar, bem como estimular o planejamento pessoal por meio de estratégias alternativas de gestão do tempo, em apenas um encontro. As oficinas assumiram o formato de uma apresentação de PowerPoint integrada com atividades baseadas na gestão do tempo.

Inicialmente, foi introduzido o tema da gestão do tempo. Foi realizada uma pergunta aberta aos participantes sobre a forma como se organizavam, com o intuito de identificar as estratégias de planejamento utilizadas e em que atividades costumavam perder mais tempo. Na sequência, buscou-se identificar os problemas enfrentados pelos participantes por meio de exemplos que constavam em uma planilha. Os participantes assinalaram suas dificuldades e, após, discutiram com o grupo para identificar as possíveis causas das mesmas.

Exemplos de dificuldades frequentes relacionadas à gestão do tempo:

- Dificuldades frequentes relacionadas à gestão do tempo

- Deixo o que tenho para fazer para a última hora.

- Adio as tarefas de hoje para o dia seguinte.

- Demoro a iniciar minhas tarefas.

- Interrompo com frequência o que estou fazendo.

- Desisto de tarefas pouco agradáveis depois de iniciá-las.

- Chego atrasado(a) aos meus compromissos.

- Faço tarefas secundárias antes das mais urgentes. 
- Planejo muitas tarefas e acabo não dando conta.

- Tenho uma agenda, mas não consigo utilizá-la.

- Quanto mais tempo tenho, menos tarefas realizo.

- Dedico meu tempo ao trabalho, prejudicando minha vida pessoal.

Após, foi proposta uma discussão aberta no grupo, na qual os participantes refletiram se as atividades em que estavam envolvidos eram importantes para seus objetivos de carreira, de forma a revisar prioridades e reorganizar o tempo dedicado às atividades que desempenham. Em seguida, os participantes receberam uma agenda (planilha com os dias da semana e horários) para preencherem com suas atividades e visualizarem as tarefas que tomavam mais tempo e se algo poderia ser modificado.

A última parte da oficina buscava oferecer dicas para a gestão do tempo, baseadas na literatura (Basso, Graf, Lima, Schmidt, \& Bardagi., 2013; Chase et al., 2013; Claessens et al., 2007; Covey, 2002; Roberts et al., 2006; Van Eerde, 2003). Foi salientado que não existe uma "receita mágica" que funcione para todas as pessoas. Daí a importância de discutir as dificuldades individuais para identificar os aspectos que cada estudante deve focalizar. Algumas estratégias foram discutidas com os participantes. A primeira delas foi a centralização das informações relativas às tarefas a serem realizadas em um só lugar, como agenda ou celular. Assim, os estudantes poderiam evitar a perda de tempo decorrente da procura por informações que não lembravam onde haviam sido armazenadas. A segunda dica foi reservar 10 minutos para planejar o dia seguinte, considerando os compromissos prioritários e o tempo de deslocamento para cada um deles. Também foi explicada a diferença entre tarefas importantes (aquelas que ainda se tem tempo para realizar) e urgentes (aquelas em que tempo para realizá-las é muito curto ou já terminou). A partir disso, foi sugerido que as pessoas priorizem as atividades importantes na sua lista de tarefas para evitar que essas se tornem urgentes. Outra sugestão foi definir horários para o lazer e para a utilização das redes sociais da internet, bem como desligar alertas sonoros ou visuais, a fim de evitar interrupções durante a realização de outras tarefas. Destacou-se, ainda, a importância de possuir horários livres para acomodar ajustes na agenda no caso de ocorrer algum imprevisto. Em relação à autodisciplina, foi sugerido que os estudantes tentassem seguir os horários de trabalho, mesmo quando desempenham atividades em casa, para que as responsabilidades não sejam acumuladas ou deixadas para trás. Por fim, foram discutidas as vantagens de realizar as tarefas com qualidade e finalizá-las antes do prazo. Neste sentido, salientaram-se os benefícios de evitar interrupções desnecessárias, de conhecer as próprias limitações e de saber dizer "não" para atividades que não são prioritárias ou de sua responsabilidade. Durante a apresentação das possíveis estratégias que podem contribuir para uma melhor gestão do tempo e das tarefas ("dicas"), os participantes foram encorajados a identificar, individualmente, quais delas poderiam auxiliá-los a lidar com as dificuldades que haviam identificado anteriormente.

A oficina foi finalizada com um vídeo sobre procrastinação (disponível em: http://www.youtube. $\mathrm{com} /$ watch?v=4P785j15Tzk). Este vídeo trata sobre uma dificuldade frequente de evitar atividades que as distraem das tarefas prioritárias.

Buscou-se tornar o encontro o mais interativo possível, de forma que os participantes pudessem expressar suas dúvidas e dificuldades sobre o tema perante o grupo. Ao final das atividades realizadas, os participantes responderam uma ficha de avaliação (sobre tópicos, desempenho dos facilitadores, atividades) com o objetivo de promover melhorias para as próximas oficinas. Também foram realizadas notas de campo, que foram analisadas posteriormente.

\section{Resultados e Discussão}

Os resultados referem-se às observações e registros obtidos durante a realização das oito oficinas. Os profissionais que ministravam cada oficina efetuavam anotações sobre suas impressões, bem como elaboravam pareceres a respeito de cada atividade promovida. Durante os encontros, procurou-se analisar alguns aspectos do trabalho realizado, como se o mesmo contemplou ou não os objetivos propostos e a receptividade dos acadêmicos frente aos recursos metodológicos utilizados nas oficinas. Ademais, foi possível avaliar as principais dificuldades manifestas pelos participantes quanto à gestão do tempo ao longo das atividades.

As oficinas possuíam dois objetivos principais: avaliar e discutir a forma como os participantes costumam se organizar no que diz respeito à gestão do 


\section{De Olineira, Clarissa Tochetto; Carlotto, Rodrigo Carvalho; Teixeira, Marco Antônio Pereira; Dias, Ana Cristina Garcia (2015). Oficinas de Gestão do Tempo com Estudantes Universitários.}

tempo e estimular o planejamento pessoal, através do ensino de estratégias de gestão do tempo. As fichas de avaliação preenchidas pelos participantes ao final da oficina possibilitaram identificar o nível de satisfação dos estudantes com o trabalho desenvolvido, de forma mais objetiva. Verificou-se que as oficinas contemplaram plenamente as expectativas dos estudantes $(84,70 \%)$, e a quase totalidade dos alunos $(97,65 \%)$ enfatizou que participaria de outra oficina oferecida pelo projeto, evidenciando a necessidade dos universitários em compartilhar suas dificuldades frente à gestão do tempo. Grande parte dos acadêmicos administrava de forma pouco eficaz seus horários e compromissos, o que com frequência afetava negativamente vários aspectos de sua vida pessoal e profissional. Para muitos, a participação na oficina representou um primeiro momento de reflexão sobre a temática. Além disso, durante os encontros, observou-se o interesse dos jovens na busca de dicas sobre o conteúdo trabalhado.

Cabe ressaltar que a maior parte dos participantes era constituída por alunos de semestres iniciais e intermediários de seus cursos, com uma média de idade de 22,8 anos. Supõe-se que os problemas enfrentados pelos acadêmicos em relação à administração do tempo, associados à necessidade de planejar a carreira profissional, motivaram a procura por essa oficina. Ademais, acredita-se que o envolvimento significativo de alunos dos anos iniciais da graduação nas oficinas esteja relacionado à necessidade desses jovens em assumir uma postura ativa frente à adaptação à universidade, marcada pela necessidade de uma maior autonomia em relação ao aprendizado e pela busca de conhecimentos e oportunidades extraclasse que, por sua vez, requerem uma gestão de tempo mais eficiente por parte dos estudantes (Soares et al., 2009). Já a demanda de estudantes formandos e de pós-graduação pode ser compreendida pela necessidade de conciliar diferentes atividades características da fase que estão vivendo (estágio, emprego, trabalho de conclusão de curso, cobrança por produção científica etc), conforme relatado pelos participantes durante as oficinas. Contudo, não se pode assumir que a menor demanda por parte de formandos e pós-graduandos, tal como constatada por esse estudo, signifique que os mesmos possuem melhor gestão do tempo, a ponto de não ter interesse em participar de uma oficina sobre essa temática, ou pior gestão do tempo, a ponto de não conseguirem participar de atividades como essa.
De maneira geral, os tópicos abordados ao longo das atividades foram bem recebidos pelos estudantes. A exposição de dicas relacionadas ao planejamento e à gestão de tempo foi o tópico que mais despertou o interesse dos acadêmicos. Muitas vezes, os universitários surpreendiam-se com a simplicidade e praticidade das dicas apresentadas. Foi enfatizada a utilização de algumas estratégias ao longo das oficinas, a saber: uso da agenda, planejamento equilibrado do dia, semana e mês, identificação das situações nas quais o estudante perde muito tempo, discernimento das tarefas importantes ou urgentes, e programação de espaços no dia a dia para lidar com imprevistos. Muitos aspectos abordados nas oficinas assemelham-se aos temas trabalhados em outros treinamentos de gestão do tempo descritos na literatura, como a identificação de problemas enfrentados pelos participantes na gestão do tempo, o estabelecimento de tarefas prioritárias e como dizer "não" de maneira assertiva para atividades que não são de responsabilidade do indivíduo (Van Eerde, 2003), ou ainda a identificação dos procedimentos adotados pelos participantes para se organizar e a elaboração da agenda semanal (Basso et al., 2013).

Ademais, pode-se perceber a receptividade dos acadêmicos frente aos recursos metodológicos utilizados. Em especial, verificou-se que a atividade desenvolvida no formato de "oficinas" propiciou uma dinâmica interessante ao trabalho. Esse modelo didático possibilitou a participação efetiva e espontânea dos estudantes, permitindo a eles compartilhar suas angústias em relação à organização do tempo. Frequentemente, as dificuldades expostas pelos membros do grupo eram semelhantes, de forma que o relato de um estudante estimulava a participação dos demais. Nesse contexto, a presença de facilitadores treinados e a apresentação de técnicas de gestão de tempo colaboraram para o bom andamento das oficinas.

Ainda, se pode verificar que os alunos que apresentavam problemas para gerir seu tempo costumavam demonstrar dificuldades em comum. Essas podem ser agrupadas em quatro categorias principais: a) comportamentos relacionados à procrastinação, por exemplo, o adiamento das responsabilidades para a "última hora", a demora em iniciar tarefas e a perda de tempo significativa nas redes sociais da internet, b) dificuldades em dizer "não" às demandas alheias, c) problemas para lidar com a carga horária de seus cursos, d) dificuldades em conciliar estu- 
dos, convivência familiar e lazer. Entre esses tópicos, a perda de tempo em redes sociais, especialmente no Facebook, foi apontada como a maior "vilã" pelos acadêmicos no que se refere a sua gestão do tempo. O número inesgotável de possibilidades oferecidas pela rede mundial de computadores, muitas vezes, interfere na definição das prioridades e na organização do tempo (Barbosa, 2011).

Outro ponto enfatizado pelos acadêmicos foi a dificuldade em dizer "não" diante de determinadas tarefas. Os jovens relataram que esse tópico interferia diretamente no processo de gestão de tempo. Em diversos casos, o desejo de mostrar-se interessado e solícito faz com que o estudante concorde em assumir demandas excessivas. Comportamentos similares foram encontrados em um estudo sobre habilidades sociais assertivas, no qual se observou que um número significativo de universitários apresentou dificuldades para manifestar sua opinião, fazer e recusar pedidos e expressar raiva (Camacho, \& Vila, 2009). Algumas estratégias que podem ajudar a dizer "não" para tarefas que não se pode assumir são: não decidir imediatamente para que se possa verificar se a atividade está de acordo com os objetivos e prioridades, agradecer a lembrança e reconhecer a importância do pedido antes de afirmar que não é a pessoa mais indicada para aquela tarefa e sugerir alguém no lugar ou que não tem condições de assumir aquele compromisso, propor assumir um papel menor do que o oferecido no desempenho da tarefa (Chase et al., 2013). Programas que estimulem o treino de habilidades sociais podem auxiliar a população universitária no enfrentamento dessas dificuldades.

Também se pode pensar que a dificuldade em gerir o tempo decorra de demandas que os estudantes não estavam acostumados até então, tais como o elevado número de avaliações, o maior grau de dificuldade das mesmas e o excesso de carga horária de alguns cursos de graduação. A dificuldade em lidar com esse novo ritmo de estudos foi manifestada várias vezes pelos participantes das oficinas. O tempo é um recurso limitado para os graduandos, uma vez que o ingresso na universidade demanda um número maior de tarefas a serem realizadas pelos acadêmicos (Couto, 2014; Sampaio, 2011). Uma rotina de avaliações, prazos mais rígidos de entrega de trabalhos e a conciliação com atividades extracurriculares são elementos que podem interferir na gestão do tempo dos acadêmicos.
Parte significativa dos alunos destinava muito tempo aos estudos e pouco tempo a atividades de lazer e convivência familiar. $\mathrm{O}$ acadêmico que não distribui de forma equilibrada seus horários e gerencia de forma equivocada seus compromissos, pode estar mais propenso a desenvolver sintomas relacionados ao estresse, ansiedade e depressão (Soares, 2008). Administrar bem o tempo consiste em fazer com que a pessoa planeje estratégias, táticas e ações de forma sistematizada, conciliando aspectos de sua vida pessoal e profissional (Augustin, 2008). Portanto, torna-se importante estabelecer um planejamento que considere essa harmonia e propicie uma melhor gestão do tempo dos indivíduos (Estrada, Flores, \& Schimith, 2011).

A execução otimizada de atividades e a redução de urgências são demandas da Era do Conhecimento. Nesse contexto, a gestão do tempo ganha destaque devido à necessidade de se obter um melhor aproveitamento do dia a dia. Essa nova configuração exige tanto o aprimoramento de hábitos, como o uso de técnicas e instrumentos para gerir as atividades pessoais, profissionais e acadêmicas com maior eficiência (Estrada et al., 2011).

\section{Considerações finais}

Buscou-se apresentar a experiência de oito oficinas de gestão do tempo voltadas a estudantes do ensino superior, descrevendo observações e registros obtidos durante a realização das atividades. As oficinas realizadas através deste projeto possibilitaram avaliar, discutir e aprimorar habilidades para o desenvolvimento de carreira de estudantes universitários, como a gestão do tempo.

O alto índice de procura dos discentes pela oficina de gestão do tempo mostra o reconhecimento da importância dessa prática por parte dos alunos da graduação e da pós-graduação. As dificuldades manifestadas pelos participantes suscitam dois pontos importantes para análise. O primeiro refere-se à necessidade de desenvolver mais atividades do gênero. $\mathrm{O}$ segundo ponto é um questionamento: por que um número considerável de jovens tem apresentado tantos problemas para organizar o seu tempo? A realização de pesquisas que investiguem os "motivos" dessa dificuldade pode subsidiar futuras intervenções junto a esse público.

As oficinas realizadas apresentaram algumas limitações. Uma delas refere-se ao local onde os primeiros encontros foram realizados, no campus da universidade, que se localiza em um bairro afastado do centro da cidade. Nem todos os estudantes pos- 


\section{De Olineira, Clarissa Tochetto; Carlotto, Rodrigo Carvalho; Teixeira, Marco Antônio Pereira; Dias, Ana Cristina Garcia (2015). Oficinas de Gestão do Tempo com Estudantes Universitários.}

suem condições para se deslocar até o campus, especialmente aqueles que trabalham durante o dia e estudam à noite ou aqueles que estudam em cursos situados no centro do município. Por isso, além de oferecer as oficinas para estudantes em geral, também foram atendidas as solicitações de PETs e ONGs formadas por universitários para realizar as oficinas na sede das mesmas, a fim de facilitar a participação de diferentes alunos. Outra limitação corresponde à duração da oficina. Os participantes manifestaram o desejo de que o encontro fosse mais longo. Entretanto, optou-se por manter uma hora e trinta minutos porque acredita-se que esse tempo seja suficiente para cumprir o objetivo da oficina sem torná-la cansativa para os participantes e para os profissionais que a conduzem. Por fim, não se realizou o acompanhamento do quanto o conteúdo da oficina foi apreendido pelos participantes e se os aspectos discutidos foram realmente implementados no dia a dia, embora $\mathrm{o}$ interesse demonstrado pelos mesmos possa indicar o desejo de incorporar os conhecimentos e estratégias trabalhados na sua rotina. Além disso, o interesse em participar da oficina, em avaliar e em discutir diversos tópicos da gestão do tempo mostram o quanto os estudantes consideram importante o desenvolvimento dessa habilidade.

Este estudo ainda apresenta contribuições para a prática profissional de psicólogos que trabalham em diferentes contextos. Os problemas relatados pelos participantes das oficinas (procrastinação, dificuldades em dizer "não" às demandas alheias, em lidar com a carga horária de seus cursos e em conciliar estudos, convivência familiar e lazer) muitas vezes não são exclusivos de estudantes universitários. Alunos de ensino fundamental e médio também podem ter comportamentos de procrastinação e, como consequência, apresentarem dificuldades na sua capacidade de organização para estudar para as avaliações e ansiedade para realizá-las. Adolescentes de ensino médio, em especial, que estão se preparando para ingressar em um curso superior, também podem sofrer com a eventual sobrecarga de estudo do colégio e de cursos preparatórios para o vestibular. Essa so- brecarga, que por si só já pode trazer prejuízos aos adolescentes, também pode comprometer o tempo dedicado à convivência familiar e ao lazer. Estudantes de pós-graduação e professores universitários também podem enfrentar esses mesmos problemas, com o agravante da pressão por produção (publicações). Adultos que trabalham em organizações ou mesmo como profissionais liberais também podem encontrar dificuldades semelhantes às apresentadas pelos participantes das oficinas. É possível que a procrastinação de atividades de trabalho, dificuldades de delegar tarefas que não são de sua responsabilidade, de não assumir mais um trabalho quando já está com a carga horária preenchida e de conciliar o tempo que possui com outros papéis que assume na vida comprometam sua produtividade, autoeficácia e bem-estar.

Psicólogos que trabalham em diferentes contextos podem se beneficiar das informações desse estudo, já que os tópicos abordados nas oficinas podem ser facilmente adaptados às necessidades do público com que trabalham. Psicólogos que trabalham em escolas podem promover oficinas semelhantes a essas, com foco em como lidar com as dificuldades vivenciadas de gestão do tempo, para que não prejudiquem os hábitos de estudo e seu desempenho nas avaliações. Psicólogos que trabalham em universidades podem replicar essas oficinas ou, ainda, utilizar as informações e técnicas relatadas em atendimentos individuais ou grupais com foco na adaptação acadêmica, no caso de estudantes de graduação, ou na produtividade, no caso de estudantes de pós-graduação e professores pesquisadores. Psicólogos que trabalham em organizações podem criar treinamentos ou oficinas similares, priorizando a habilidade de delegar tarefas e de manter o foco em reuniões de equipe para aumentar a produtividade. Por último, mas não menos importante, a leitura desse estudo também pode ser útil para psicólogos clínicos, uma vez que dificuldades na gestão do tempo podem ser um dos fatores que impedem os pacientes/clientes de desempenharem suas responsabilidades ou de implementarem mudanças como gostariam. 


\section{Referências}

Augustin, E. S. (2008) Planejamento pessoal como base e ligação para o planejamento estratégico organizacional (Dissertação de Mestrado). Programa de Pós-Graduação em Engenharia de Produção, Universidade Federal de Santa Maria, Santa Maria.

Barbosa, C. (2011). A tríade do tempo. Rio de Janeiro, RJ: Sextante.

Basso, C., Graf, L. P., Lima, F. C., Schmidt, B., \& Bardagi, M. P. (2013). Organização de tempo e métodos de estudo: Oficinas com estudantes universitários. Revista Brasileira de Orientação Profissional, 14(2), 277-288. Recuperado de http://pepsic.bvsalud.org/scielo.php?pid=S1679-33902013000200012\&script=sci_arttext

Camacho, A., \& Vila, E. M. (2009). Levantamento de variáveis antecedentes e consequentes que favoreceram a aprendizagem e a manutenção de dificuldades interpessoais em universitários. Semina: Ciências Biológicas e da Saúde, 30(1), 47-66. doi: 10.5433/1679-0367.2009v30n1p47

Chase, J. D., Topp, R., Smith, C. E., Cohen, M. Z., Fahrenwald, N., Zerwic, J. J. ...Conn, V. S. (2013). Time management strategies for research productivity. Western Journal of Nursing Research, 35(2) 155-176. doi: 10.1177/0193945912451163

Claessens, B. J. C., Van Eerde, W., Rutte, C. G., \& Roe, R. A. (2007). A review of the time management literature. Personnel Review, 36(2), 255-276. doi: 10.1108/00483480710726136

Couto, M. C. P. P. (2014). Como manejar o tempo na academia. In S. H. Koller, M. C. P. P. Couto, \& J. V. Hohendorff(Orgs.), Manual de produção científi$c a$ (pp. 167-177). Porto Alegre, RS: Penso.

Covey, S. R. (2002). Os 7 hábitos das pessoas altamente eficazes (12a ed.). São Paulo, SP: Best Seller.

Estrada, R. J. S., Flores, J. T., \& Schimith, C. D. (2011). Gestão do tempo como apoio ao planejamento estratégico pessoal. Revista de Administração da Universidade Federal de Santa Maria, 4(2), 315-332. Recuperado de http://cascavel.ufsm.br/revistas/ ojs-2.2.2/index.php/reaufsm/article/view/3349

Figueiredo, F. J. (2008). Como ajudar os alunos a estudar e a pensar?: Auto-regulação da aprendizagem. Revista Millenium, 34, 233-258. Recuperado de http://repositorio.ipv.pt/handle/10400.19/377

Guerreiro-Casanova, D. C., \& Polydoro, S. A. J. (2011). Autoeficácia na formação superior: Percepções durante $\mathrm{o}$ primeiro ano de graduação.
Psicologia: Ciência e Profissão, 31(1), 50-65. doi: 10.1590/S1414-98932011000100006

König, C. J., \& Kleinmann, M. (2007). Time management problems and discounted utility. The Journal of Psychology, 141(3), 321-334. doi: 10.3200/JRLP.141.3.321-336

MacCann, C., Fogarty, G., \& Roberts, R. D. (2012). Strategies for success in vocational education: Time management is more important for part-time than fulltime students. Learning and Individual Differences, 22(2), 618-623. doi:10.1016/j.lindif.2011.09.015

Roberts, R. D., Schulze, R., \& Minsky, J. (2006). The relation of time management dimensions to scholastic outcomes. Annual Meeting of the American Educational Research Association, San Francisco, CA.

Rosário, P., Nunes, T., Magalhães, C., Rodrigues, A.,Pinto, R., \& Ferreira, P. (2010). Processos de auto-regulação da aprendizagem emalunos com insucesso no $1^{\circ}$ ano de universidade. Psicologia Escolar Educacional, 14(2), 349-358. doi: 10.1590/S1413-85572010000200017

Sampaio, R. K. N. (2011). Procrastinação acadêmica e autorregulação da aprendizagem em estudantes universitários (Dissertação de Mestrado). Faculdade de Educação, Universidade Estadual de Campinas, Campinas, São Paulo.

Sarriera, J. C., Paradiso, Â. C., Schütz, F. F., \& Howes, G. P. (2012). Estudo comparativo da integração ao contexto universitário entre estudantes de diferentes instituições. Revista Brasileira de Orientação Profissional, 13(2), 163-172. Recuperado de http:// pepsic.bvsalud.org/scielo.php?pi$\mathrm{d}=$ S1679-33902012000200004\&script=sci_arttext

Soares, A. B., Poubel, L. N., \& Mello, T. V. S. (2009). Habilidades sociais e adaptação acadêmica: Um estudo comparativo em instituições de ensino público e privado. Aletheia, (29), 27-42. Recuperado de http://pepsic.bvsalud.org/scielo.php?pi$\mathrm{d}=$ S1413-03942009000100004\&script=sci_arttext

Soares, S. A. (2008). Gestão do tempo e da comunicação. Mediação, 8(7), 20-27. Recuperado de http://www. fumec.br/revistas/mediacao/article/view/277

Van Eerde, W. (2003). Procrastination at work and time management training. The Journal of Psychology, 137(5), 421-434. doi: 10.1080/00223980309600625

Zampetakis, L. A., Bouranta, N., \& Moustakis, V. S. (2010). On the relationship between individual creativity and time management. Thinking Skills and Creativity, 5(1), 23-32. doi: 10.1016/j. tsc.2009.12.00110.1016/j.tsc.2009.12.001 
De Olineira, Clarissa Tochetto; Carlotto, Rodrigo Carvalho; Teixeira, Marco Antônio Pereira; Dias, Ana Cristina Garcia (2015). Oficinas de Gestão do Tempo com Estudantes Universitários.

\section{Clarissa Tochetto de Oliveira}

Mestre em Psicologia pela Universidade Federal de Santa Maria, Santa Maria - RS. Brasil.

E-mail: clarissa.tochetto@gmail.com

\section{Rodrigo Carvalho Carlotto}

Mestre em Psicologia pela Universidade Federal de Santa Maria, Santa Maria - RS. Brasil.

E-mail: carlotto@jc.iffarroupilha.edu.br

\section{Marco Antônio Pereira Teixeira}

Doutorado em Psicologia pela Universidade Federal do Rio Grande do Sul, Porto Alegre - RS. Brasil. Docente da Universidade Federal do Rio Grande do Sul, Porto Alegre - RS. Brasil.

E-mail: mapteixeira@yahoo.com.br

\section{Ana Cristina Garcia Dias}

Doutorado em Psicologia Escolar e do Desenvolvimento pela Universidade de São Paulo, São Paulo - SP. Brasil. Docente da Universidade federal de Santa Maria, Santa Maria - RS. Brasil.

E-mail: anacristinagarciadias@gmail.com

\author{
Endereço para envio de correspondência: \\ Universidade Federal do Rio Grande do Sul, Instituto \\ de Psicologia, Rua Ramiro Barcelos, 2600. Centro. \\ CEP: 90035-003. Porto Alegre - RS. Brasil.
}

Recebido 30/10/2014

Aprovado 15/12/2015

Received 10/30/2014

Approved 12/15/2015

Recibido 30/10/2014

Aceptado 15/12/2015

Como citar: Oliveira, C. T., Carlotto, R. C., Teixeira, M. A. P., \& Dias, A. C. G. (2016). Oficinas de gestão do tempo com estudantes universitários. Psicologia: Ciência e Profissão, 36(1): 224-233. doi:10.1590/1982-3703001482014

How to cite: Oliveira, C. T., Carlotto, R. C., Teixeira, M. A. P., \& Dias, A. C. G. (2016). Workshops on time management with college students. Psicologia: Ciência e Profissão, 36(1):224-233. doi:10.1590/1982-3703001482014

Cómo citar: Oliveira, C. T., Carlotto, R. C., Teixeira, M. A. P., \& Dias, A. C. G. (2016). Talleres de gestión del tiempo con estudiantes universitarios. Psicologia: Ciência e Profissão, 36(1): 224-233. doi:10.1590/1982-3703001482014 